\title{
Expression of a Novel Cadherin in the Mouse and Human Intestine
}

\author{
DEDRICK E. MOULTON, WALLACE CRANDALL, RUPAL LAKHANI, AND MARK E. LOWE \\ Department of Pediatrics [D.E.M., W.C., R.L., M.E.L.], Department of Pharmacology and Molecular \\ Biology [M.E.L.], Washington University School of Medicine, St. Louis, Missouri 63110, U.S.A.
}

\begin{abstract}
ABST
Members of the cadherin superfamily mediate critical inter-
actions in tissue differentiation and organogenesis, including
differentiation and maintenance of the intestine. In this study, we
report the identification and expression of a novel cadherin in the
intestinal epithelium. We identified this cDNA by subtraction
hybridization and obtained subsequent clones by screening a
human cDNA library. Tissue distribution of the mRNA encoding
the cadherin was assessed by RNA blot, reverse transcriptase
PCR, and in situ hybridization. Protein expression was analyzed
by protein blot and immunohistochemistry. The cDNA encodes
an integral membrane protein with four consecutive cadherin
binding domains followed by a series of mucin domains, a
unique feature of this cadherin. Differences in the mucin domains
account for four splice-forms. Multiple potential SH3-binding
domains and a single potential PDZ-binding domain follow the
\end{abstract}
The intestinal epithelium undergoes a rapid and continuous process of cellular renewal involving cell turnover and regeneration $(1,2)$. Cell turnover entails a sequence of cell proliferation, differentiation, migration, and programmed cell death. Pluripotential epithelial stem cells reside near the base of each crypt of Lieberkuhn and provide a continuous supply of mature epithelial cells. Daughter cells that arise from these stem cells proliferate rapidly and differentiate during a bipolar migration into one of four principle epithelial cell lineages. As a result, enterocytes, goblet cells, and enteroendocrine cells populate the villus epithelium whereas Paneth cells reside at the base of the crypts along with stem cells $(3,4)$. Cell lineages undergo differentiation as they migrate up the epithelial villus. After 3-5 d, cells on the villous tip are shed through a process involving apoptosis (5-7). Consequently, development and maintenance of normal intestinal epithelial morphology re-

Received June 6, 2003; accepted January 22, 2004.

Correspondence: Mark E. Lowe, M.D., PhD., Children's Hospital of Pittsburgh, Pediatric Gastroenterology, 3705 5th Ave. at DeSoto St., Pittsburgh, PA 15213, U.S.A., e-mail: Mark.Lowe@chp.edu

Supported by grant DK 52574 from the National Institute of Diabetes and Digestive and Kidney Diseases.

DOI: 10.1203/01.PDR.0000125260.46861.32 transmembrane domain. Analysis revealed expression in the liver, kidney, and intestine. Three splice variants were found in the embryonic intestine as early as embryonic d 13 and in the adult intestine. The mRNA localizes to the mature enterocytes throughout the mouse small intestine and the protein, including several species from 90 to $100 \mathrm{kD}$, resides on the enterocyte basolateral membrane. We have identified intestinal expression of a novel cell cadherin with features suggesting the potential to transduce signals from neighboring cells to the cytoplasm. (Pediatr Res 55: 927-934, 2004)

Abbreviation
RT, reverse transcriptase
E, embryonic day

quires tight regulatory control of cell proliferation, migration, and differentiation along the crypt-villus axis.

The coordination of these processes in building the normal architecture of the intestinal epithelium depends on selective interactions of the cells with other cells and with the extracellular matrix $(8,9)$. Multiple molecules have been identified that mediate intercellular and cell-to-matrix adhesive functions (10-12). Most belong to one of a few supergene families, the selectin, the immunoglobulin, the integrin, or the cadherin families. Members of the integrin and cadherin families play important roles in intestinal morphogenesis and maintenance of normal epithelial villus structures $(11,13-21)$. Cadherins help maintain intestinal epithelial architecture and function by influencing migration, differentiation, and apoptosis of enterocytes. Although the intestine expresses several members of the cadherin family, E-cadherin is the predominant cell adhesion molecule in the intestinal epithelium (22).

In this study, we report the expression of a unique member of the cadherin family in the intestine as well as in liver and kidney. Tissues in the fetus also express mRNA encoding this protein at early developmental stages. In the intestine, the protein is predominately expressed in the villus epithelium and not in crypt cells. Analysis of the predicted amino acid sequence suggests that this protein is an integral membrane 
protein with four repeated sequences that are homologous to the cadherin binding domains. Unlike, classical cadherins, a tandem repeat homologous to mucin domains precedes the membrane-spanning region and the predicted cytoplasmic tail contains a proline-rich region potentially capable of binding to proteins that contain an SH3 domain. The predicted structure of this protein makes it capable of mediating interactions between maturing enterocytes in ways that may influence the architecture and function of the intestinal epithelium.

\section{MATERIALS AND METHODS}

Animal husbandry. Animal care and use were in accordance with institutional Animal Studies Committee guidelines and our animal protocol was approved by the institutional Animal Studies Committee. Mice were maintained on standard rodent chow and water ad libitum.

cDNA isolation. We isolated the original clone from a subtraction library prepared from AR42J cells (ATCC CRL1492, American Type Culture Collection, Manassas, VA, U.S.A.), a line derived from rat pancreas (23). The cells were grown in the presence and absence of dexamethasone as previously described (24). Total RNA was prepared using RNAzole according to the manufacturer's instructions (Tel Test B, Friendswood, TX, U.S.A.). Subtractive hybridization of mRNA isolated from untreated and dexamethasone treated AR42J cells was performed with the PCR-Select Subtraction Kit according to the manufacturer's standard protocol (CLONTECH, Palo Alto, CA, U.S.A.). Differentially expressed clones were sequenced by the dideoxynucleotide method using the Big Dye protocol. Each sequence was analyzed with Basic Local alignment and Search Tool for Nucleotides (BLASTN).

The novel clone isolated from the subtraction library was used as a probe of a commercially available human cDNA library, HUCL (Stratagene, Cedar Creek, TX, U.S.A.). The probe was labeled with ${ }^{32} \mathrm{P}$ by the Prime-It $\mathrm{RmT}$ random primer method according to instructions (Stratagene). Hybridization was done at $65^{\circ} \mathrm{C}$ with ExpressHyb (CLONTECH). Searching the Genbank EST database with both the human and rat clones identified additional clones. These were obtained and the nucleotide sequence of the insert determined.

$\boldsymbol{R N A}$ blot. For some analysis, multiple expression tissue arrays and multiple tissue Northern blots were screened (CLONTECH). For other analysis, total RNA was isolated from selected mouse tissues by the RNAzole method (Tel Test B). The RNA was separated by denaturing agarose gel electrophoresis and transferred to a membrane as previously described (24). Radiolabeled probes were prepared by the MAXIscript method (Ambion, Austin, TX, U.S.A.). The blots were hybridized in ULTRhyb solution according to the manufacturer's protocol (Ambion). The blots were developed on a Molecular Dynamics Storm 840 phosphoimager connected to a computer containing ImageQuaNT software (Molecular Dynamics, Sunnyvale, CA, U.S.A.).

RT-PCR analysis. Pregnant Swiss-Webster mice were obtained from Taconic Farms (Germantown, NY, U.S.A.). The pups were removed by cesarean section at E10, E13, E15, and E17. RNA was isolated by the RNAzole method using the manufacturer's protocol (Tel Test B). Primers that flanked the region encoding the mucin repeats were selected. The sequence of the downstream primer is 5'-AAC AGC ACT GCA CCT AGC CCC CGC CCA A-3' and of the upstream primer is 5' CCG CTG TTG TTG AGA TCC AGG TGT CAG-3'. RT-PCR amplification was performed with the Access RT-PCR System (Promega, Madison, WI, U.S.A.). Amplification was performed using the following parameters: $60 \mathrm{~s}$ at $95^{\circ} \mathrm{C}$ for one cycle followed by $60 \mathrm{~s}$ at $95^{\circ} \mathrm{C}, 90 \mathrm{~s}$ at $58^{\circ} \mathrm{C}, 120 \mathrm{~s}$ at $72^{\circ} \mathrm{C}$ for 2 min for 37 cycles, and 5 min at $72^{\circ} \mathrm{C}$ for 1 cycle using a Stratagene Robocycler Gradient 96. The PCR products were separated on a $2 \%$ agarose gel and stained with ethidium bromide. Each PCR product was isolated from the gel with the QIAquick Gel Extraction Kit (QIAGEN, Valencia, CA, U.S.A.) and cloned into pT-Adv vector (CLONTECH). The sequence of each band was determined by the Big-Dye method.

In situ hybridization. Mice were deeply anesthetized with $2.5 \%$ avertine and were transcardially perfused with diethlpyrocarbonate-treated PBS (DEPC-PBS) followed by $4 \%$ paraformaldehyde in DEPC-PBS. Isolated intestines were postfixed in $4 \%$ paraformaldehyde for $24 \mathrm{~h}$, followed by immersion in $10 \%$ sucrose in DEPC-PBS. The fixed tissue was embedded in OCT (Sakura Finetek USA, Inc., Torrance, CA, U.S.A.). Tenmicrometer sections were cut on a cryostat and thaw mounted onto Superfrost plus slides (Fisher Scientific, Pittsburgh, PA, U.S.A.). The original rat clone was radiolabeled with $\alpha-{ }^{33} \mathrm{P}-$ UTP and T7 polymerase with the MAXIscript kit according to the manufacturer's instructions (Ambion). The labeled probe was hybridized to the sections at an annealing temperature of $55^{\circ} \mathrm{C}$. The slides were emulsion dipped with LM-1 emulsion (Amersham Pharmacia Biotech, Little Chalfont, Buckinghamshire, U.K.) and developed according the manufacturer's directions after a 1-wk exposure. The slides were viewed with dark-field microscopy.

Antibody production. To produce an antibody, the original AR42J clone was ligated into $\mathrm{pBAD} / \mathrm{HisA}$ and a fusion protein expressed in Escherichia coli as described by the manufacturer (Invitrogen, Carlsbad, CA, U.S.A.). The fusion protein was isolated by affinity chromatography according to the method in the XPress system Protein Purification kit (Invitrogen). A polyclonal antibody was produced in rabbits. The antibody specifically recognized the recombinant protein by immunoblot analysis.

Immunoblot analysis. The entire small intestine was removed from adult mice and divided into three approximately equal parts, termed proximal, middle, and distal small intestine. Each section was extracted with SDS sample buffer and homogenized with a polytron for $60 \mathrm{~s}$ in the presence of protease inhibitors (Mini-Complete, Roche Molecular Biochemicals, Summerville, NJ, U.S.A.). An aliquot of each sample was analyzed by immunoblot after separation of the proteins by SDS-PAGE as previously described (25).

Immunofluorescence. Immunofluorescence was done as previously described except that the secondary antibody was a FITC-anti-rabbit $\operatorname{IgG}$ and the hydrogen peroxide wash was eliminated (26). The FITC-anti-rabbit IgG was incubated on the tissue sections in PBS-T $(137 \mathrm{mM} \mathrm{NaCl}, 2.7 \mathrm{mM} \mathrm{KCl}, 4.3$ 
$\mathrm{mM} \mathrm{Na}_{2} \mathrm{HPO}_{4} \cdot \mathrm{H}_{2} \mathrm{O}, 1.4 \mathrm{mM} \mathrm{KH} \mathrm{PO}_{4}, 0.2 \%$ Tween-20) for $1 \mathrm{~h}$ at room temperature. The sections were washed three times with PBS-T and then viewed under a fluorescent microscope. Controls included incubations with preimmune sera and with $100 \mu \mathrm{g} / \mathrm{mL}$ of recombinant antigen in the solution of the primary antibody.

\section{RESULTS}

Isolation of $\mu$-protocadherin clone. AR42J cells, a cell line derived from rat pancreas that represents a tissue culture cell model for pancreatic acinar cells, becomes more acinar-like after treatment with dexamethasone. Dexamethasone slows cell division, promotes cell adhesion, and increases the expression of acinar cell specific exocrine proteins and hormone receptors. We hypothesized that dexamethasone-regulated genes in AR42J cells would also mediate differentiation of acinar cells in the pancreas. To identify dexamethasoneregulated genes in AR42J cells, we performed subtractive hybridization of mRNA isolated from AR42J cells treated with dexamethasone and mRNA isolated from untreated cells. A number of differentially regulated clones were identified, but most had well-described functions and dexamethasone regulation of their expression had already been documented. Two of the isolated clones did not match any sequence in DNA databases at the time they were isolated. One clone, which was stimulated by dexamethasone, encoded an open reading frame that predicted a 257 amino acid sequence. The predicted amino acid sequence contained four regions of homology to the calcium-binding domains of cadherins.

To isolate a full-length cDNA, we used our fragment to screen a human multi-tissue cDNA library and obtained two clones. Restriction digest analysis of the clones showed that the inserts were of different sizes. One contained a $2.7-\mathrm{kb}$ insert and the other a $1.7-\mathrm{kb}$ insert. We sequenced both strands of each clone. Alignment of the sequences revealed that they were identical except that the shorter clone contained less $5^{\prime}$ sequence and had a 688-bp gap near the 3' end of the clones. Both inserts contained open reading frames, with the longer form encoding 845 amino acids and the shorter clone encoding 651 amino acids. The predicted amino acid sequences were identical; however, a gap had to be introduced into the shorter insert to optimally align the sequences. The sequences suggested that the clones represent alternatively spliced variants of the same gene.

While this work proceeded, several matching clones were deposited into databases. One clone, a novel member of the cadherin family, was isolated from rat kidney (AF221952) and named $\mu$-protocadherin $(27,28)$. This clone matched the nucleotide sequence of our original rat clone and had $74 \%$ identity to our longer human clone. Two other unpublished sequences matched our two human clones. These two clones were $2.9 \mathrm{~kb}$ (AF258676) and $2.0 \mathrm{~kb}$ (AF276242). Both of these clones contained more 5'-untranslated sequence than our clones, but they matched our long and short clones in $99 \%$ of the overlapping sequences. A third human clone deposited in GenBank (AF258675) contained $2.0 \mathrm{~kb}$ and matched the other two clones over the first $1886 \mathrm{bp}$, but had a unique sequence over the remaining 3 ' region. In addition, we identified several homologous mouse EST clones. Two were obtained and sequenced. One clone (AA869195) was about $0.8 \mathrm{~kb}$ and sequence analysis indicated that it was a partial clone. The other clone (AI747122) was about 2.9-kb long and was sequenced completely. The clone matched the rat $\mu$-protocadherin sequence (AF221952) in 90\% of the overlapping sequence, but required a 91-bp gap to optimize the alignment. BLASTN searches with these cDNA clones did not reveal any significant homology to other cDNA clones indicating that these clones represent a novel branch of the cadherin family.

Predicted protein structure. To identify protein motifs in the predicted amino acid sequence of the isolated clone, we performed computer-based domain searches (Fig. 1). Examination of the amino acid sequence of each clone showed that all of the sequences included a signal peptide. The program predicted a cleavage site between Ala25 and Gln26 for the human clones and between Ala28 and Gln29 for the rodent clones. The first of four predicted cadherin extracellular domains immediately followed the signal peptide. A single transmembrane domain was predicted for each of the clones. Between the last cadherin domain and the transmembrane domain reside 3.5 simple tandem repeats. These serine-, threonine-, and proline-rich sequences have homology to motifs found in mucins. The mouse clone only contained 2.5 mucin repeats and the entire region encoding the tandem repeats was missing from the shorter human clone. Additionally, in the mouse, a third isoform was found that lacked the linker region between the cadherin domain and the transmembrane domain as well as part of the first serine-threonine-proline rich tandem repeats (See below). A short cytoplasmic tail followed the transmembrane domain. Evaluation of the putative cytoplasmic domain revealed a proline-rich region capable of interacting with SH3 domains on other proteins, one conserved protein kinase $\mathrm{C}$ phosphorylation site (Ser-Gly-Lys) and a potential PDZ binding domain (X-Thr/Ser-Y-Val/Ile) (29). The shortest human clone (AF258675) was the exception to this pattern, as this clone did not contain any of the features found in the cytoplasmic tails of the other clones. Importantly, this clone did not contain the mucin repeats, the transmembrane domain, or the

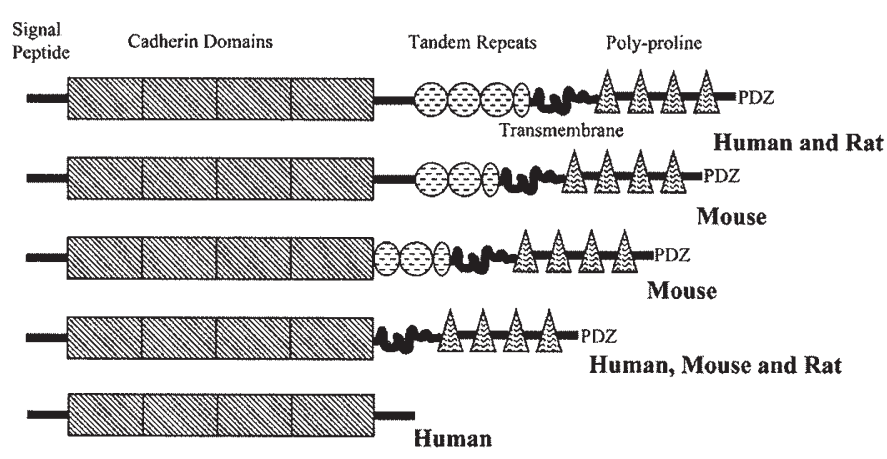

Figure 1. Schematic depiction of the predicted protein structure of the $\mu$-protocadherin isoforms. Signal P v1.1, TopPred 2, and SAPS programs were used to search for protein motifs. The cadherin domains are represented by cross-hatched boxes, the mucin repeats by circles and ovals containing dotted lines, the transmembrane domain by a free-form line, and the poly-proline domain by triangles with wavy lines. 
poly-proline regions and it may represent a secreted form of $\mu$-protocadherin.

Tissue-specific and temporal expression of $\mu$-protocadherin $\boldsymbol{m} \boldsymbol{R} \boldsymbol{N} \boldsymbol{A}$. We next determined the tissue distribution of the mRNA encoding $\mu$-protocadherin. A cDNA probe derived from our original rat clone was hybridized to multiple tissue arrays of mouse mRNA and human mRNA (Fig. 2). A strong signal was present in mouse and human small intestine, human large intestine, and in mouse and human liver and kidney. The cadherin probe also hybridized to mRNA isolated from mouse prostate and to mRNA isolated from 7-d and 17-d mouse embryos, but not to mRNA isolated from 11-d and 15-d mouse embryo. Surprisingly, mouse pancreas gave no signal even with longer exposures, whereas human pancreas gave only a faint signal.

We confirmed the presence of mRNA encoding this novel protein in mouse and human liver, kidney, and intestine by RNA blot analysis. In the mouse, all three tissues contained two mRNA species that hybridized to the $\mu$-protocadherin probe (Fig. $3 A$ ). In the human, the kidney, and liver showed three mRNA species whereas small intestine had predominantly the larger mRNA (Fig. 3B). Longer exposures revealed the two smaller species in the small intestine and the larger species in the colon (data not shown).

Contrary to our findings in the human and mouse, Goldberg et al. (27) found that rat liver did not express any mRNA encoding $\mu$-protocadherin when they hybridized the blot with a probe from the mucin domain. To determine whether speciesspecific differences in gene expression account for this observation, we hybridized a rat multiple tissue RNA blot from the same commercial source reported in the rat $\mu$-protocadherin study with our rat probe encompassing the cadherin domain of the cDNA. Using that probe, signal was apparent in the kidney but not in the liver (Fig. 4). Additionally, our probe, derived from the cadherin region, detected three bands in the rat kidney compared with the two bands previously reported when a probe derived from the mucin region was used. We confirmed the absence of mRNA in rat liver by hybridizing our probe to a blot of total rat liver RNA prepared in our laboratory and found no signal (data not shown).

We next determined whether other mucin variants were present in the mouse intestine and whether the expression pattern varied with development. To accomplish this analysis, we performed RT-PCR on total RNA isolated from fetal, suckling, and adult mouse small intestine. The PCR primers were complementary to sequence at the 3 '-end of the last cadherin repeat and to sequence just $5^{\prime}$ of the transmembrane domain. Consequently, the primers amplified the region between the cadherin repeats and the transmembrane domain, which contains linker sequence as well as the mucin repeats. Our analysis revealed three distinct bands of about 550, 270, and $80 \mathrm{bp}$ (Fig. 5). Sequence analysis of these bands showed that the larger, 550-bp band corresponded to our current clone with 2.5 mucin repeats. The 270-bp band derived from an mRNA species that has only a single mucin repeat. The smallest product derives from an mRNA homologous to the short human clone, which does not contain any of the mucin
Mouse Tissues

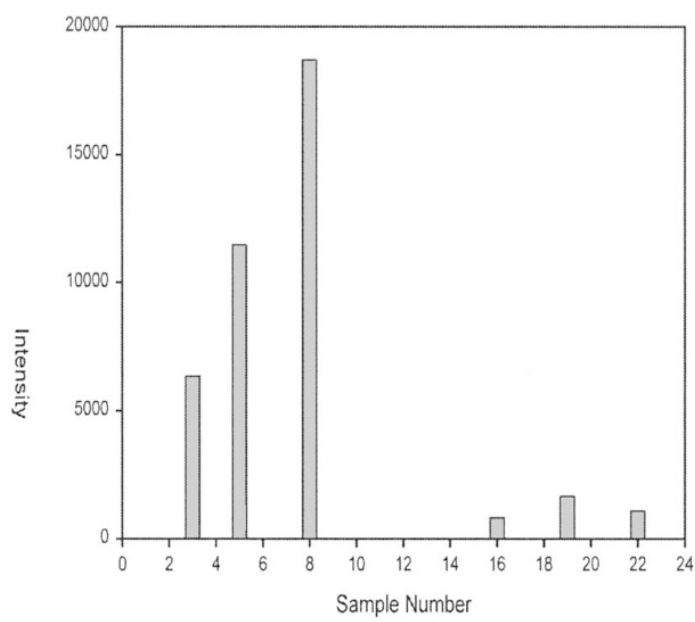

Human Tissues

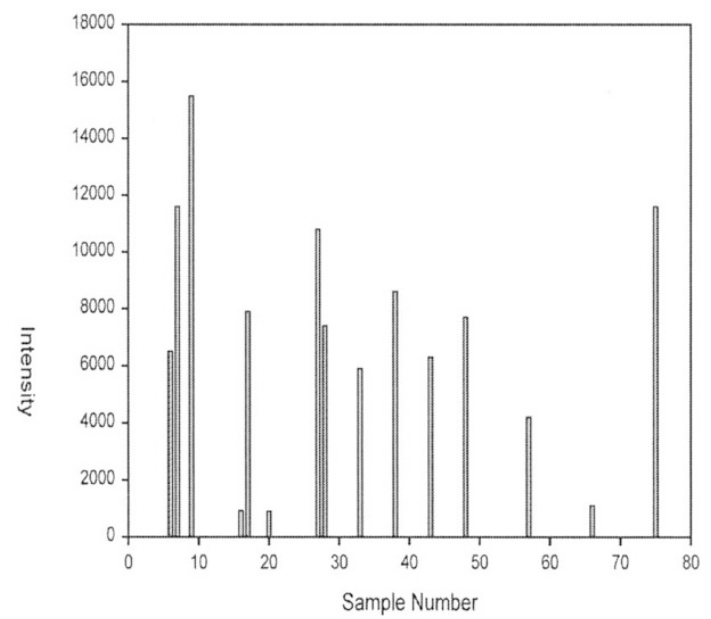

Figure 2. Multi-tissue dot blot analysis of mouse and human tissues. The mouse blot was hybridized to a probe derived from the original rat clone and the human blot was hybridized to a $1.5 \mathrm{~kb}$ restriction fragment of the long human isoform as described in Methods. Both probes spanned the cadherin domains. Tissues in bold type hybridized to the probe. The mouse tissues are: 1) brain; 2) eye; 3) liver; 4) lung; 5) kidney; 6) heart; 7) skeletal muscle; 8) small intestine; 9) pancreas; 10) thyroid; 11) thymus; 12) submaxillary gland; 13) spleen; 14) testis; 15) ovary; 16) prostate; 17) epididymis; 18) uterus; 19) embryo 7 d; 20) embryo 11 d; 21) embryo $15 \mathrm{~d}$; 22) embryo $17 \mathrm{~d}$. The human tissues are: 1) whole brain; 2) cerebellum, left; 3) substantia nigra; 4) heart; 5) esophagus; 6) transverse colon; 7) kidney; 8) lung; 9) liver; 10) leukemia HL-60; 11) fetal brain; 12) cerebral cortex; 13) cerebellum, right; 14) accumbens nucleus; 15) aorta; 16) stomach; 17) descending colon; 18) skeletal muscle; 19) placenta; 20) pancreas; 21) HeLa S3; 22) fetal heart; 23) frontal lobe; 24) corpus callosum; 25) thalamus; 26) left atrium; 27) duodenum; 28) rectum; 29) spleen; 30) bladder; 31) adrenal gland; 32) leukemia K0562; 33) fetal kidney; 34) parietal lobe; 35) amygdala; 36) pituitary gland; 37) right atrium; 38) jejunum; 39) thymus; 40) uterus; 41) thyroid gland; 42) leukemia MOLT-4; 43) fetal liver; 44) occipital lobe; 45) caudate nucleus; 46) spinal cord; 47) left ventricle; 48) ileum; 49) peripheral blood leukocyte; 50) prostate; 51) salivary gland; 52) Burkett's lymphoma, Raji; 53) fetal spleen; 54) temporal lobe; 55) hippocampus; 56) right ventricle; 57) ileocecum; 58) lymph node; 59) testis; 60) mammary gland; 61) Burkett's lymphoma, Daudi; 62) fetal thymus; 63) paracentral gyrus of cerebral cortex; 64) medulla oblongata; 65) interventricular septum; 66) appendix; 67) bone marrow; 68) ovary; 69) colorectal adenocarcinoma SW480; 70) fetal lung; 71) pons; 72) putamen; 73) apex of the heart; 74) ascending colon; 75) trachea; 76) lung carcinoma A549. 


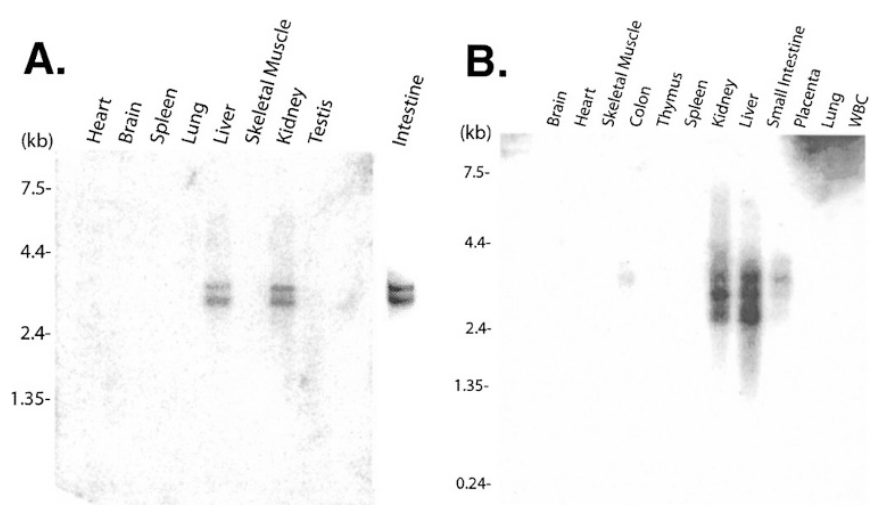

Figure 3. RNA blots of total RNA isolated from mouse and human tissues. (A) A commercially available RNA blot of various mouse tissues and an RNA blot of mouse intestine prepared in this lab were probed with the original rat clone as described in "Methods." $(B)$ A commercially available human RNA blot was probed with a $1.5 \mathrm{~kb}$ restriction fragment of the long human isoform as described in "Methods."

(kb)

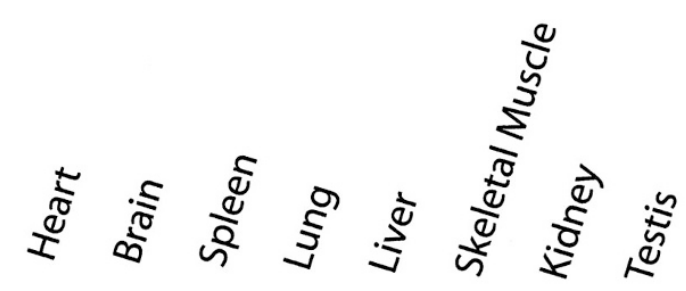

$7.5-$

4.4-

2.4-

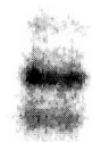

$1.35-$

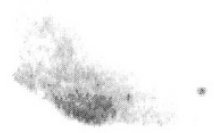

Figure 4. RNA blot of total RNA isolated from rat tissues. A commercially available RNA blot was probed with the original rat clone as described in "Methods."

repeats. All three bands were present at every developmental stage examined.

Cell-specific expression of $\mu$-protocadherin. To identify the intestinal cells that express mRNA for this clone we performed in situ hybridization. Both anti-sense and sense probes were made from a 300-bp fragment of our rat clone. We hybridized the probes to sections of adult small intestine. The anti-sense probe strongly hybridized to intestinal epithelial cells whereas the sense probe showed only background signal (Fig. 6). The

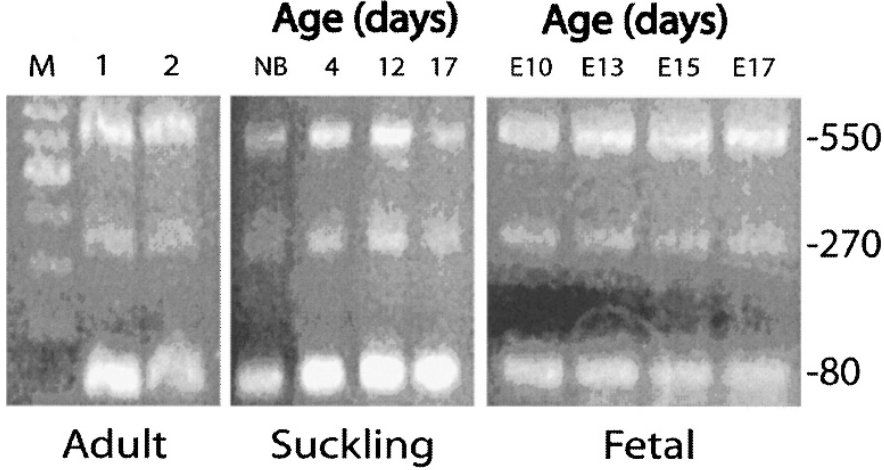

Figure 5. Analysis of $\mu$-protocadherin isoforms at various developmental stages by RT-PCR. Total RNA was isolated from the small intestine at various developmental stages and RT-PCR was performed as described in "Methods." The developmental stage is given below each panel. $M, 100$ bp markers; $N B$, newborn.

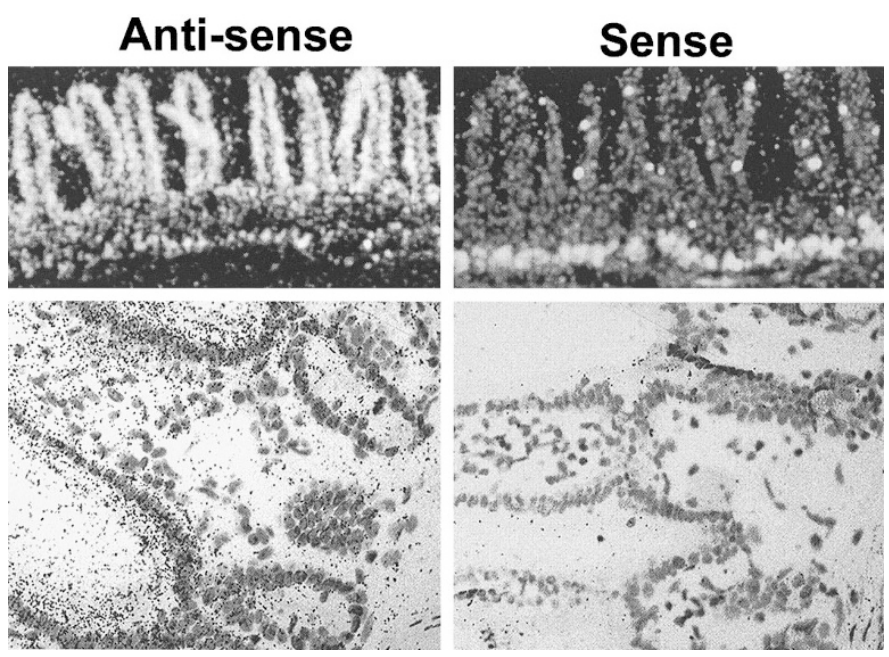

Figure 6. In situ hybridization of mouse small intestine. Frozen sections of mouse small intestine were hybridized to antisense or sense riboprobes prepared from the original rat clone. The upper panels show dark-field images of the intestinal mucosa. The lower panels show bright-field images of the base of the villus and the crypts.

mature epithelial cells lining the villi had the most intense concentration of grains. The signal seen in the region of the crypts in the dark-field sections (Fig. 6, upper panels) was birefringence from the Paneth cells and was present in both sense and anti-sense sections. At higher magnification, the crypts had little to no signal (Fig. 6, lower panels). Identical patterns were found when we performed in situ hybridization of small intestine from newborn, 7-, 14-, and 23-d-old pups (data not shown).

Expression of $\mu$-protocadherin in small intestine by immunoblot. We next determined whether the intestine synthesizes $\mu$-protocadherin protein. We generated an antibody to the cadherin domain of the partial rat clone. We then used the antibody as a probe of protein blots and for immunohistochemistry. For protein blots, we either processed the entire small intestine or sectioned the small intestine into thirds and homogenized each sample in SDS PAGE sample buffer. The solubilized proteins were separated by SDS PAGE and transferred to a membrane (Fig. 7). Duplicate samples were stained 


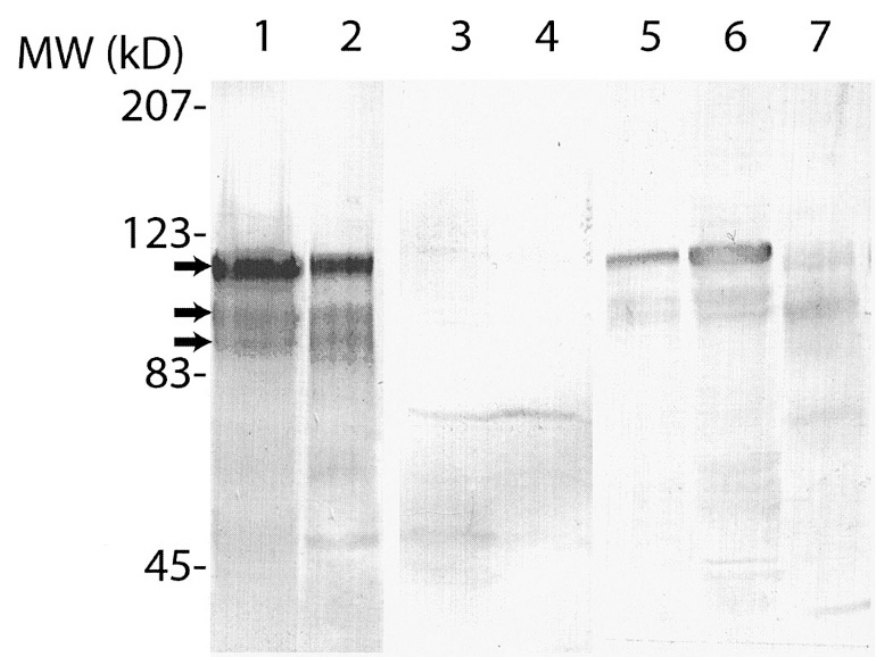

Figure 7. Protein blots of extracts from adult mouse small intestine. Extracts of mouse small intestine were prepared and the proteins separated by SDSPAGE as described in "Methods." Lanes 1 and 2, two separate extracts of whole small intestine. Lanes 3 and 4, samples in lanes 1 and 2 stained with preimmune serum. Lane 5, distal third of the small intestine. Lane 6, middle third of the small intestine. Lane 7, proximal third of the small intestine.

with $\mu$-protocadherin antisera or preimmune sera. The $\mu$-protocadherin antibody detected one predominant band of about $100-110 \mathrm{kD}$, which is larger than the predicted molecular mass of $91 \mathrm{kD}$ for the longest isoform and suggests that $\mu$-protocadherin is posttranslationally modified, most likely, by glycosylation. Two other faster migrating bands were also detected. The distribution of the protein varied in the rostral to caudal axis. The bands, particularly the upper band, were more robustly expressed in the distal and middle sections than in the proximal small intestine. In contrast, the preimmune sera did not detect any proteins. In other experiments, neither the upper band nor the two lower bands were detected if recombinant antigen was included in the incubation with the primary antibody against $\mu$-protocadherin (data not shown).

Expression of $\mu$-protocadherin in small intestine by immunofluorescence. We stained sections of small intestine with our antibody to identify the location of $\mu$-protocadherin in the intestinal epithelial cell. In sections of distal adult small intestine, we found specific staining of villus enterocytes (Fig. 8, A and $B$ ). In cross-section, the staining appeared mostly on the lateral borders (Fig. $8 A$ ). The intensity of the staining increased along the lateral borders as enterocytes approach the apical extrusion zone of the villus and there is apparent apical staining at the villus tip (Fig. 8B). Staining was light to undetectable in sections of the distal small intestine (data not shown). No staining was apparent with preimmune serum and recombinant antigen blocked binding of the antibody (Fig. 8, $C$ and $D$ ).

\section{DISCUSSION}

We have identified and begun to characterize novel members of the cadherin superfamily, $\mu$-protocadherin, which are expressed in developing and adult small intestine. These proteins have unique structural features that distinguish it from other family members and that provide clues to the function of $\mu$-protocadherin. The presence of cadherin repeats in the pre-
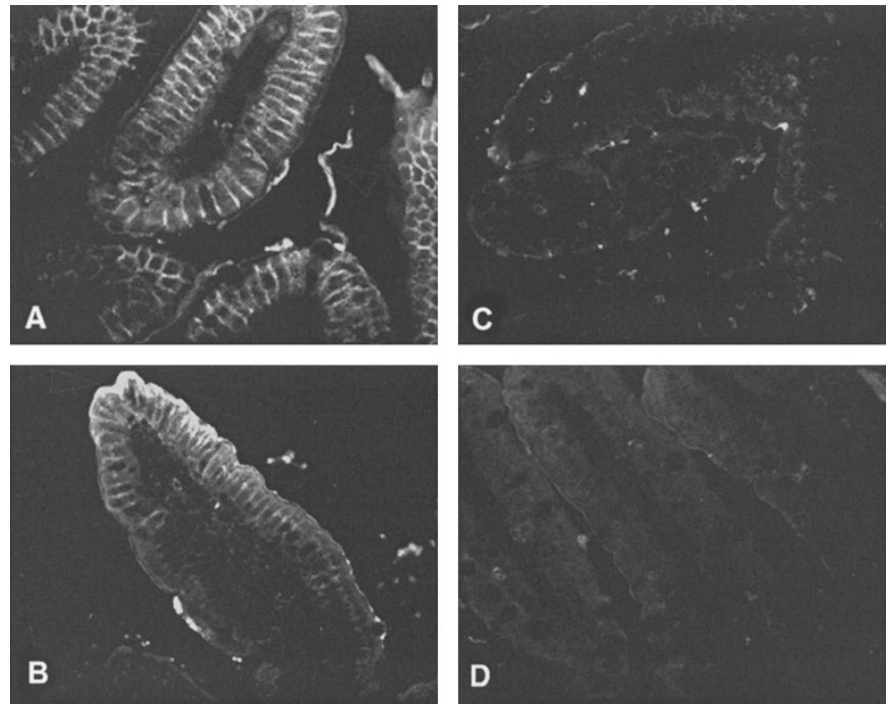

Figure 8. Immunohistochemistry of adult mouse small intestine. Paraffinembedded sections were stained with anti- $\mu$-protocadherin antiserum followed by fluorescein-conjugated anti-rabbit IgG antiserum as described in "Methods." ( $A$ and $B$ ) Villi stained with anti- $\mu$-protocadherin antiserum. $(C)$ preimmune sera replaced the anti- $\mu$-protocadherin antiserum. $(D)$ In the incubation with the anti- $\mu$-protocadherin antiserum, $100 \mu \mathrm{g} / \mathrm{mL}$ of recombinant antigen was included.

dicted amino acid sequence of $\mu$-protocadherin suggests that these are adhesion molecules. An adhesive function is supported by the location of $\mu$-protocadherin in regions of contact between intestinal epithelial cells, as shown by immunohistochemistry. Further support for an adhesive function of $\mu$-protocadherins was provided by a study that showed enhanced aggregation of $\mathrm{L}$ cells transfected with the long form of $\mu$-protocadherin (27).

Multiple isoforms of the $\mu$-protocadherins result from differential splicing of the mucin-like domains, which distinguish the $\mu$-protocadherins from other cadherin family members (27). Two isoforms, one with 3.5 mucin repeats and one with no repeats, were previously identified in rat kidney and as human clones deposited in GenBank. In this article, we report two additional isoforms found in mouse small intestine. One of these isoforms has 2.5 mucin repeats. The other has one 2.25 mucin repeats and does not contain the linker region between the mucin repeats and the cadherin domains. The 3.5 mucin repeat isoform has not been found in mouse as yet. Our RNA blots of human and rat tissues suggest that a third isoform may exist in these species. The third band in rat kidney is larger than the previously described isoforms. This larger band may represent another mRNA species with a cadherin domain apart from the $\mu$-protocadherin family, but is unlikely to be a described cadherin because nucleotide sequence searches of GenBank with our probe sequence only identified $\mu$-protocadherin sequences and revealed no significant homology with other known cadherins. The number of mucin repeats can affect the function of the $\mu$-protocadherins by modifying cadherinmediated adhesion, by providing a specific binding site for adhesins, or by serving as ligand binding sites.

Other structural features of the $\mu$-protocadherins may contribute to the function of these novel proteins. All, but one of 
the isoforms contain a transmembrane domain. In these isoforms, the putative cytoplasmic tail possesses structural motifs that implicate the $\mu$-protocadherins in signal transduction. Specifically, the putative intracellular region contains a potential protein kinase $\mathrm{C}$ phosphorylation site, a PDZ-binding domain, and four poly-proline repeats that can potentially bind to $\mathrm{SH} 3$ domains. These domains have been implicated in signal transduction, which often requires the assembly of signaling proteins into protein complexes at the plasma membrane (2932). Phosphorylation of tyrosine or serine frequently regulates the assembly of these signaling complexes. Complex formation is accomplished through the interactions of modular protein binding domains, such as PDZ and SH3 domains. Therefore, the structure of the $\mu$-protocadherins suggests they have the capability to form adhesive interactions that induce signaling events.

The function of the $\mu$-protocadherins in various tissues may also be determined by the temporal pattern of isoform expression during development. Expression of the two isoforms detected in the adult rat kidney by Goldberg et al. (27) was independently regulated during development. Only the longest isoform was found in the fetal rat kidney and both isoforms were present in the adult kidney. A different expression pattern occurs in the small intestine of the mouse. We detected three isoforms in the small intestine as early as E10 of gestation. This expression pattern persisted throughout gestation and did not change after birth. In contrast, we only detected two mRNA species in RNA blots of mouse intestine. The disparity between the PCR and RNA blot results has several possible explanations. One of the species could be present in low abundance and not detected by RNA blot. Alternatively, alterations in the untranslated regions of the mRNA could result in similar sized products despite the differences in the coding regions. Protein blots of intestine also revealed three immunoreactive species. The results do not distinguish between differential glycosylation of the same isoform and the expression of three different isoforms in the intestine. Still, the results from the Goldberg et al. study and this study suggest that expression of the $\mu$-protocadherin isoforms can be differentially regulated during development of dissimilar tissues.

$\mu$-Protocadherin is a novel member of the cadherin superfamily with unique structural features that implicate it in cell signaling and cell adhesion. The gene encoding $\mu$-protocadherin (MUCDHL) is located on human chromosome $11 \mathrm{p} 15.5$ and mouse chromosome 7 (33). The expression of several splice variants can potentially provide a broad range of function through regulation of the amount expressed and the intracellular location of the various isoforms. Given these properties, $\mu$-protocadherins are well suited to influence the development and architecture of the intestinal epithelium, which is a dynamic tissue requiring regulated differentiation, cell proliferation, cell migration, and programmed cell death to develop and maintain its normal architecture. Adhesive events, many modulated by known cadherins, contribute critically to these fundamental processes of epithelial cell biology. The need for a detailed understanding about the types of cadherins involved in adhesive events, the function of each cadherin, the regulation of cadherin function during development, and tissue maintenance provides a rich area for research with widereaching implications. Dysfunction of $\mu$-protocadherin could play a role in tissue injury and repair in multiple diseases of the bowel such as Crohn disease or ulcerative colitis. The central role of cadherins in epithelial cell biology implies the potential for developing novel treatment, management, and prevention methods for chronic ulcerative and inflammatory lesions of the intestinal epithelium.

\section{REFERENCES}

1. Loeffler M, Birke A, Winton D, Potten C 1993 Somatic mutation, monoclonality and stochastic models of stem cell organization in the intestinal crypt. J Theor Biol 160:471-491

2. Schmidt GH, Wilkinson MM, Ponder BA 1985 Cell migration pathway in the intestinal epithelium: an in situ marker system using mouse aggregation chimeras. Cell 40:425-429

3. Bry L, Falk P, Huttner K, Ouellette A, Midtvedt T, Gordon JI 1994 Paneth cell differentiation in the developing intestine of normal and transgenic mice. Proc Natl Acad Sci U S A 91:10335-10339

4. Cheng H, Leblond CP 1974 Origin, differentiation and renewal of the four main epithelial cell types in the mouse small intestine. I. Columnar cell. Am J Anat 141:461-479

5. Wright NA, Al-Nafussi A 1982 The kinetics of villus cell populations in the mouse small intestine. II. Studies on growth control after death of proliferative cells induced by cytosine arabinoside, with special reference to negative feedback mechanisms. Cell Tissue Kinet 15:611-621

6. Hall PA, Coates PJ, Ansari B, Hopwood D 1994 Regulation of cell number in the mammalian gastrointestinal tract: the importance of apoptosis. J Cell Sci 107:35693577

7. Gavrieli Y, Sherman Y, Ben-Sasson SA 1992 Identification of programmed cell death in situ via specific labeling of nuclear DNA fragmentation. J Cell Biol 119:493-501

8. Jawhari A, Farthing M, Pignatelli M 1997 The importance of the E-cadherin-catenin complex in the maintenance of intestinal epithelial homoeostasis: more than intercellular glue? Gut 41:581-584

9. Perry I, Hardy R, Tselepis C, Jankowski JA 1999 Cadherin adhesion in the intestinal crypt regulates morphogenesis, mitogenesis, motogenesis, and metaplasia formation. Mol Pathol 52:166-168

10. Yap AS, Brieher WM, Gumbiner BM 1997 Molecular and functional analysis of cadherin-based adherens junctions. Ann Rev Cell Dev Biol 13:119-146

11. Gumbiner BM 1996 Cell adhesion: the molecular basis of tissue architecture and morphogenesis. Cell 84:345-357

12. Gumbiner BM 2000 Regulation of cadherin adhesive activity. J Cell Biol 148:399404

13. Behrens J, Birchmeier W, Goodman SL, Imhof BA 1985 Dissociation of MadinDarby canine kidney epithelial cells by the monoclonal antibody anti-arc-1: mechanistic aspects and identification of the antigen as a component related to uvomorulin. J Cell Biol 101:1307-1315

14. McNeill H, Ozawa M, Kemler R, Nelson WJ 1990 Novel function of the cell adhesion molecule uvomorulin as an inducer of cell surface polarity. Cell 62:309-316

15. Nagafuchi A, Shirayoshi Y, Okazaki K, Yasuda K, Takeichi M 1987 Transformation of cell adhesion properties by exogenously introduced E-cadherin cDNA. Nature 329:341-343

16. Frixen UH, Nagamine Y 1993 Stimulation of urokinase-type plasminogen activator expression by blockage of E-cadherin-dependent cell-cell adhesion. Cancer Res 53:3618-3623

17. Gumbiner B, Stevenson B, Grimaldi A 1988 The role of the cell adhesion molecule uvomorulin in the formation and maintenance of the epithelial junctional complex. J Cell Biol 107:1575-1587

18. Jongen WM, Fitzgerald DJ, Asamoto M, Piccoli C, Slaga TJ, Gros D, Takeichi M, Yamasaki H 1991 Regulation of connexin 43-mediated gap junctional intercellular communication by $\mathrm{Ca} 2+$ in mouse epidermal cells is controlled by E-cadherin. J Cell Biol 114:545-555

19. Watabe M, Nagafuchi A, Tsukita S, Takeichi M 1994 Induction of polarized cell-cell association and retardation of growth by activation of the E-cadherin-catenin adhesion system in a dispersed carcinoma line. J Cell Biol 127:247-256

20. Hermiston ML, Gordon JI 1995 Organization of the crypt-villus axis and evolution of its stem cell hierarchy during intestinal development. Am J Physiol 268:G813-G822

21. Hermiston ML, Gordon JI 1995 In vivo analysis of cadherin function in the mouse intestinal epithelium: essential roles in adhesion, maintenance of differentiation, and regulation of programmed cell death. J Cell Biol 129:489-506

22. Hermiston ML, Wong MH, Gordon JI 1996 Forced expression of E-cadherin in the mouse intestinal epithelium slows cell migration and provides evidence for nonautonomous regulation of cell fate in a self-renewing system. Genes Dev 10:985-996

23. Christophe J 1994 Pancreatic tumoral cell line AR42J: an amphicrine model. Am J Physiol 266:G963-G971

24. Kullman J, Gisi C, Lowe ME 1996 Dexamethasone-regulated expression of pancreatic lipase and two related proteins in AR42J cells. Am J Physiol 270:G746-G751

25. Lowe ME, Strauss AW 1990 Expression of a Nagao-type, phosphatidylinositolglycan anchored alkaline phosphatase in human choriocarcinomas. Cancer Res 50:3956-3962 
26. Lowe ME 1992 Site-specific mutations in the $\mathrm{COOH}$-terminus of placental alkaline phosphatase: a single amino acid change converts a phosphatidylinositol-glycananchored protein to a secreted protein. J Cell Biol 116:799-807

27. Goldberg M, Peshkovsky C, Shifteh A, Al-Awqati Q 2000 mu-Protocadherin, a nove developmentally regulated protocadherin with mucin-like domains $\mathrm{J}$ Biol Chem 275:24622-24629

28. Goldberg M, Wei M, Tycko B, Falikovich I, Warburton D 2002 Identification and expression analysis of the human mu-protocadherin gene in fetal and adult kidneys. Am J Physiol Renal Physiol 283:F454-F463

29. Pawson T, Scott JD 1997 Signaling through scaffold, anchoring, and adaptor proteins Science 278:2075-2080
30. Fanning AS, Anderson JM 1999 PDZ domains: fundamental building blocks in the organization of protein complexes at the plasma membrane. J Clin Invest 103:767772

31. Kay BK, Williamson MP, Sudol M 2000 The importance of being proline: the interaction of proline-rich motifs in signaling proteins with their cognate domains. FASEB J 14:231-241

32. Webb BL, Hirst SJ, Giembycz MA 2000 Protein kinase C isoenzymes: a review of their structure, regulation and role in regulating airways smooth muscle tone and mitogenesis. Br J Pharmacol 130:1433-1452

33. Goldberg M, Wei M, Yuan L, Murty VV, Tycko B 2003 Biallelic expression of HRAS and MUCDHL in human and mouse. Hum Genet 112:334-342 